GOET-TP 100/95

December 1995

\title{
Integrable Discretizations of Chiral Models via Deformation of the Differential Calculus
}

\author{
Aristophanes Dimakis and Folkert Müller-Hoissen \\ Institut für Theoretische Physik \\ Bunsenstr. 9, D-37073 Göttingen, Germany
}

\begin{abstract}
A construction of conservation laws for chiral models (generalized $\sigma$-models) on a two-dimensional space-time continuum using differential forms is extended in such a way that it also comprises corresponding discrete versions. This is achieved via a deformation of the ordinary differential calculus. In particular, the nonlinear Toda lattice results in this way from the linear (continuum) wave equation. The method is applied to several further examples. We also construct Lax pairs and Bäcklund transformations for the class of models considered in this work.
\end{abstract}

\section{Introduction}

Some years ago we observed that a certain deformation of the ordinary calculus of differential forms on $\mathbb{R}^{n}$ can be used to discretize classical continuum field theories [1]. For this purpose one has to formulate the theory in terms of differential forms. The deformation of the differential calculus then induces a corresponding deformation of the theory built on it. In particular, the Wilson loop formulation of lattice gauge theory originates in this way from continuum Yang-Mills theory. In this letter we present another application of the method, a discretization of chiral models (or generalized $\sigma$-models) preserving complete integrability. After a brief introduction to deformations of the ordinary differential calculus on $\mathbb{R}^{2}$ we generalize the derivation of conservation laws given for chiral models in [3]. As an example, we then derive the nonlinear Toda lattice [4] from the continuum wave equation. Further examples illustrate the method and reveal its limitations. We also construct Lax pairs for the discretized chiral models and present Bäcklund transformations.

\section{Deformation of the ordinary differential calculus on $\mathbb{R}^{2}$}

In the ordinary differential calculus on manifolds, functions commute with differentials. It is possible, however, to dispense with this property while keeping the familiar rules for

\footnotetext{
${ }^{1}$ See [2] and the references cited there.
} 
the exterior derivative (see [1] for details). The latter are consistent with the following commutation relations,

$$
\mathrm{d} t f(t, x)=f(t+\tau, x) \mathrm{d} t, \quad \mathrm{~d} x f(t, x)=f(t, x+\lambda) \mathrm{d} x
$$

where $\tau$ and $\lambda$ are real parameters, $f$ is a function on $\mathbb{R}^{2}$ and $t, x$ are the canonical coordinate functions on $\mathbb{R}^{2}$. In particular,

$$
[\mathrm{d} t, t]=\tau \mathrm{d} t, \quad[\mathrm{~d} t, x]=[\mathrm{d} x, t]=0, \quad[\mathrm{~d} x, x]=\lambda \mathrm{d} x .
$$

Using these relations we obtain for $\tau, \lambda \neq 0$

$$
\mathrm{d} f=\left(\partial_{+t} f\right) \mathrm{d} t+\left(\partial_{+x} f\right) \mathrm{d} x=\mathrm{d} t\left(\partial_{-t} f\right)+\mathrm{d} x\left(\partial_{-x} f\right)
$$

with

$$
\partial_{+x} f(t, x)=\frac{1}{\lambda}[f(t, x+\lambda)-f(t, x)], \quad \partial_{-x} f(t, x)=\frac{1}{\lambda}[f(t, x)-f(t, x-\lambda)]
$$

and similar expressions for $\partial_{ \pm t} f(t, x)$. This shows that for $\tau, \lambda \neq 0$ the differential calculus is actually defined on the algebra $\mathcal{A}$ of all real functions on $\mathbb{R}^{2}$. The deformed differential calculus has more 'constants' than the ordinary one. From the above formulas one finds that $\mathrm{d} f=0$ if and only if $f(t+\tau, x)=f(t, x)$ and $f(t, x+\lambda)=f(t, x)$. Let us choose a point, say 0 , in $\mathbb{R}$ and construct the ideal $\mathcal{I}_{\tau}$ of $\mathcal{A}$ generated by $f_{\tau}(t, x)-f_{\tau}(0, x)$ for all functions $f_{\tau} \in \mathcal{A}$ which are periodic (with period $\tau$ ) in the first argument. Then the algebra $\mathcal{A} / \mathcal{I}_{\tau}$ is isomorphic to the algebra of real-valued functions on $\mathbb{Z} \times \mathbb{R}$. If $\mathcal{I}_{\tau, \lambda}$ denotes the ideal of functions generated by $f_{\tau, \lambda}(t, x)-f_{\tau, \lambda}(0,0)$ for all functions periodic in both arguments, one finds that $\mathcal{A} / \mathcal{I}_{\tau, \lambda}$ is isomorphic to the algebra of real-valued functions on $\mathbb{Z}^{2}$.

In what follows, depending on whether the parameters $\tau$ and $\lambda$ are zero or not, $M$ denotes either $\mathbb{R}^{2}, \mathbb{R} \times \mathbb{Z}, \mathbb{Z} \times \mathbb{R}$, or $\mathbb{Z}^{2}$. Correspondingly, let $\mathcal{A}$ denote the algebra of real functions $f(t, x)$ which are smooth in both arguments, or functions $f_{k}(t):=f(t, k \lambda)$ smooth in the first argument, respectively functions $f_{k}(x):=f(k \tau, x)$ smooth in the second argument, or the algebra of all real functions $f_{k}(n):=f(n \tau, k \lambda)$ on $\mathbb{Z}^{2}$. The differential calculus defined above is then a differential calculus on the algebra $\mathcal{A}$. Acting with the exterior derivative on (2) we obtain the 2 -form relations

$$
\mathrm{d} x \mathrm{~d} x=0, \quad \mathrm{~d} t \mathrm{~d} x+\mathrm{d} x \mathrm{~d} t=0, \quad \mathrm{~d} t \mathrm{~d} t=0 .
$$

Hence $\mathrm{d} t \mathrm{~d} x$ is a basis of the space of 2 -forms $\Omega^{2}$ as a left or right $\mathcal{A}$-module. There are no forms of higher grade, i.e., $\Omega^{k}=\{0\}$ for $k \geq 3$. Let $\Omega:=\bigoplus_{k \geq 0} \Omega^{k}$ denote the differential algebra (where $\Omega^{0}=\mathcal{A}$ ).

We introduce an inner product $():, \Omega \times \Omega \rightarrow \mathcal{A}$ via $(f, g)=f g$ for $f, g \in \mathcal{A}$,

$$
(\mathrm{d} t, \mathrm{~d} t)=-1, \quad(\mathrm{~d} x, \mathrm{~d} x)=1, \quad(\mathrm{~d} t, \mathrm{~d} x)=0, \quad(\mathrm{~d} t \mathrm{~d} x, \mathrm{~d} t \mathrm{~d} x)=-1
$$

and

$$
(\psi, \phi)=(\phi, \psi), \quad(\psi, f \phi)=f(\psi, \phi)
$$

\footnotetext{
${ }^{2}$ In order to perform the limit $\tau \rightarrow 0$ (or $\lambda \rightarrow 0$ ) we have to restrict $\mathcal{A}$ to those functions which are differentiable in $t$ (respectively, $x$ ).
} 
for $\psi, \phi \in \Omega$. If $^{2} \psi$ and $\phi$ have different grades, then $(\psi, \phi)$ is set to zero. As a consequence of these definitions we find

$$
(\psi f, \phi)=(\psi, \phi f)
$$

A Hodge $*$-operator can now be introduced as an $\mathbb{R}$-linear operator on $\Omega$ via

$$
(\mathrm{d} t \mathrm{~d} x, \phi * \psi):=-(\phi, \psi)
$$

It satisfies the relations

$$
*(\psi f)=f * \psi, \quad \psi * \phi=\phi * \psi
$$

where $\phi$ and $\psi$ must have the same grade, and

$$
* 1=\mathrm{d} t \mathrm{~d} x, \quad * \mathrm{~d} t=-\mathrm{d} x, \quad * \mathrm{~d} x=-\mathrm{d} t, \quad *(\mathrm{~d} t \mathrm{~d} x)=-1 .
$$

Furthermore,

$$
* * \psi(t, x)=(-1)^{r+1} \psi(t-\tau, x-\lambda)
$$

for $\psi \in \Omega^{r}$.

The notion of an integral generalizes to our deformed differential calculus in a natural way [1]. In the following sections we only need to consider one-dimensional integrals. It is therefore sufficient here to define the integral for functions on $\mathbb{Z}$. An indefinite integral is indeed determined by

$$
\mathrm{d} \int^{x} f\left(x^{\prime}\right) \mathrm{d} x^{\prime}=f(x) \mathrm{d} x
$$

up to a 'constant', i.e., a periodic function. A corresponding definite integral is then only defined over unions of intervals which are integer multiples of $\lambda$. One obtains

$$
\int_{m \lambda}^{n \lambda} f(x) \mathrm{d} x=\lambda \sum_{k=m}^{n-1} f(k \lambda)
$$

where $m, n \in \mathbb{Z}, n>m(\operatorname{cf} \llbracket$ 迎.

\section{Chiral models and conservation laws in two dimen- sions}

In this section we essentially follow Brezin et al [3]. However, the following not only works for the ordinary differential calculus but also for its deformations considered in the previous section. $\mathcal{B}$ denotes a finite dimensional algebra of matrices and $\mathcal{B}^{*}$ the group of invertible elements of $\mathcal{B}$. Let $g: M \rightarrow \mathcal{B}$ be invertible, i.e., $g(t, x) \in \mathcal{B}^{*}$ for all $t, x$. In terms of

$$
A:=g^{-1} \mathrm{~d} g
$$

\footnotetext{
${ }^{3}$ Our inner product corresponds to a metric with Lorentzian signature. The formalism works as well with a Euclidean metric.
} 
the field equations of a chiral model (generalized $\sigma$-model) are

$$
\mathrm{d} * A=0 \text {. }
$$

The conservation laws of such a model are obtained inductively as follows. Let $\Gamma$ be the space of fields $\Psi: M \rightarrow \mathcal{B}$ and $D: \Gamma \rightarrow \Omega^{1} \otimes_{\mathcal{A}} \Gamma$ the exterior covariant derivative given by

$$
D \Psi=\mathrm{d} \Psi+A \Psi
$$

Since $A$ is a 'pure gauge' we have

$$
F:=\mathrm{d} A+A A=0 .
$$

Moreover, using (16) and (10) we find

$$
\mathrm{d} *\left(A^{i}{ }_{j} \Psi^{j}\right)=\mathrm{d}\left(\Psi^{j} * A^{i}{ }_{j}\right)=\left(\mathrm{d} \Psi^{j}\right) * A^{i}{ }_{j}=A^{i}{ }_{j} * \mathrm{~d} \Psi^{j}
$$

and thus

$$
\mathrm{d} * D \Psi=D * \mathrm{~d} \Psi
$$

Let $J^{(m)}: M \rightarrow \Omega^{1} \otimes \mathcal{B}$ be a conserved current, i.e.,

$$
\mathrm{d} * J^{(m)}=0
$$

Since the first cohomology group of $M$ is trivial, i.e., $H^{1}(M)=\{0\}$, there exists $\chi^{(m)}$ : $M \rightarrow \mathcal{B}$ such that

$$
J^{(m)}=* \mathrm{~d} \chi^{(m)}
$$

Then

$$
J^{(m+1)}:=D \chi^{(m)}
$$

is also conserved since

$$
\mathrm{d} * J^{(m+1)}=\mathrm{d} * D \chi^{(m)}=D * \mathrm{~d} \chi^{(m)}=D J^{(m)}=D D \chi^{(m-1)}=F \chi^{(m-1)}=0 .
$$

The induction starts with $\chi^{(0)}=I$, the unit matrix. We then obtain an infinite number of conserved charges given by

$$
Q^{(m)}:=\int_{t=\mathrm{const}} * J^{(m)}
$$

Let us calculate the first two of them. We have $J^{(1)}=D I=A$ and therefore

$$
Q^{(1)}=\int_{t=\mathrm{const}} * A=-\int A_{0}(t-\tau, x) \mathrm{d} x
$$

where $A=A_{0} \mathrm{~d} t+A_{1} \mathrm{~d} x$. Since $* \mathrm{~d} \chi^{(1)}=J^{(1)}=A$ we find $\mathrm{d} \chi^{(1)}=* A(t+\tau, x+\lambda)$ by use of (12). Hence

$$
\chi^{(1)}(t, x)=-\int^{x} A_{0}\left(t, x^{\prime}+\lambda\right) \mathrm{d} x^{\prime}
$$


and

$$
* J^{(2)}=* D \chi^{(1)}=J^{(1)}+*\left(A \chi^{(1)}\right) .
$$

Using (10) we find

$$
Q^{(2)}=\int_{t=\mathrm{const}} * J^{(2)}=\int A_{1}(t, x) \mathrm{d} x-\int A_{0}(t-\tau, x) \chi^{(1)}(t, x) \mathrm{d} x .
$$

Let us introduce

$$
\chi:=\sum_{m=0}^{\infty} \gamma^{m} \chi^{(m)}
$$

where $\gamma$ is a parameter. From (22) and (23) we obtain

$$
* \mathrm{~d} \chi^{(m+1)}=D \chi^{(m)} \text {. }
$$

Multiplying by $\gamma^{m+1}$ and summing over $m$ leads to

$$
* \mathrm{~d} \chi=\gamma D \chi
$$

The field equations (16) are integrability conditions of the linear system (32). This is seen as follows. Acting with $D$ on (32) and using $F=0$, we find $D * \mathrm{~d} \chi=0$. Applying $*$ to (32) we get $\mathrm{d} \chi(t-\tau, x-\lambda)=\gamma * D \chi$ and thus $\mathrm{d} * D \chi=0$ which, together with $D * \mathrm{~d} \chi=0$, implies $\mathrm{d} * A=0$. Introducing $J:=* \mathrm{~d} \chi$ we have

$$
Q(t):=\int_{t=\mathrm{const}} * J=\int_{t=\mathrm{const}} \mathrm{d} \chi(t-\tau, x-\lambda)=\left.\chi(t-\tau, x)\right|_{-\infty} ^{+\infty} .
$$

\section{Examples}

1. Toda lattice. Let $\mathcal{B}$ be the algebra $\mathbb{R}$ of real numbers and let us write $g=e^{-q}$ with a function $q: M \rightarrow \mathbb{R}$. The field equations (16) then read

$$
\mathrm{d} *\left(e^{q} \mathrm{~d} e^{-q}\right)=0 \text {. }
$$

(a) $\tau=\lambda=0$. Then (34) is just the wave equation $\mathrm{d} * \mathrm{~d} q=0$, respectively,

$$
\partial_{t}^{2} q-\partial_{x}^{2} q=0
$$

(b) $\tau=0$ and $\lambda \neq 0$. Then

$$
A=-\dot{q}_{k} \mathrm{~d} t+\frac{1}{\lambda}\left(e^{q_{k}-q_{k+1}}-1\right) \mathrm{d} x, \quad * A=\dot{q}_{k} \mathrm{~d} x-\frac{1}{\lambda}\left(e^{q_{k-1}-q_{k}}-1\right) \mathrm{d} t
$$

where $\dot{q}_{k}:=d q_{k} / d t$. The field equations become

$$
\ddot{q}_{k}=\frac{1}{\lambda^{2}}\left[e^{q_{k-1}-q_{k}}-e^{q_{k}-q_{k+1}}\right]
$$


which are those of the nonlinear Toda lattice [4]. The conserved charges can be obtained using the method described in the previous section. In particular, evaluation of (26) using (14) leads to

$$
Q^{(1)}=\lambda \sum_{k=-\infty}^{\infty} \dot{q}_{k}
$$

which is the total momentum. Furthermore,

$$
\chi^{(1)}(t, k \lambda)=\lambda \sum_{\ell=-\infty}^{k} \dot{q}_{\ell}(t)
$$

(modulo addition of a constant) and thus

$$
Q^{(2)}=\sum_{k=-\infty}^{\infty}\left[e^{q_{k}-q_{k+1}}-1+\lambda^{2} \sum_{\ell \leq k} \dot{q}_{k} \dot{q}_{\ell}\right] .
$$

This yields

$$
Q^{(2)}-\frac{1}{2}\left[Q^{(1)}\right]^{2}=\sum_{k}\left[\frac{1}{2} \lambda^{2} \dot{q}_{k}^{2}+e^{q_{k}-q_{k+1}}-1\right]
$$

which is the total energy.

(c) $\tau \neq 0, \lambda \neq 0$. Then

$$
\begin{aligned}
A & =\frac{1}{\tau}\left(e^{q_{k}(n)-q_{k}(n+1)}-1\right) \mathrm{d} t+\frac{1}{\lambda}\left(e^{q_{k}(n)-q_{k+1}(n)}-1\right) \mathrm{d} x \\
* A & =-\frac{1}{\tau}\left(e^{q_{k}(n-1)-q_{k}(n)}-1\right) \mathrm{d} x-\frac{1}{\lambda}\left(e^{q_{k-1}(n)-q_{k}(n)}-1\right) \mathrm{d} t
\end{aligned}
$$

and the field equations are

$$
\frac{1}{\tau^{2}}\left[e^{q_{k}(n-1)-q_{k}(n)}-e^{q_{k}(n)-q_{k}(n+1)}\right]=\frac{1}{\lambda^{2}}\left[e^{q_{k-1}(n)-q_{k}(n)}-e^{q_{k}(n)-q_{k+1}(n)}\right] .
$$

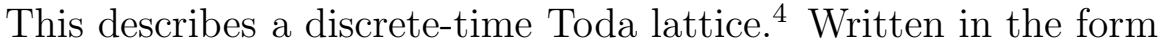

$$
e^{q_{k}(n)-q_{k}(n+1)}=e^{q_{k}(n-1)-q_{k}(n)}-\frac{1}{c^{2}}\left[e^{q_{k-1}(n)-q_{k}(n)}-e^{q_{k}(n)-q_{k+1}(n)}\right]
$$

with $c:=\lambda / \tau$, we see that the rhs is not necessarily positive in contrast to the lhs. As a consequence, there is a constraint on initial values. Exact solutions are given by

$$
q_{k}(n)=f(k+n)-2 k \ln c
$$

where $f$ is an arbitrary function. These 'left movers' do not have a right-moving counterpart. From (42) and (23) it is evident that $q_{k}(n)$ enters the conserved charges only through the quantities

$$
U_{k}(n):=e^{q_{k}(n)-q_{k+1}(n)}, \quad V_{k}(n):=e^{q_{k}(n)-q_{k}(n+1)}
$$

\footnotetext{
${ }^{4}$ See also [5] and references given there for a class of discrete time generalized Toda lattices.
} 
for which we obtain the following first order system,

$$
\begin{aligned}
U_{k}(n+1) & =\frac{V_{k+1}(n)}{V_{k}(n)} U_{k}(n), \\
V_{k}(n+1) & =V_{k}(n)+c^{-2}\left[U_{k}(n+1)-U_{k-1}(n+1)\right] .
\end{aligned}
$$

The first conserved charge is

$$
Q^{(1)}(n)=c \sum_{k}\left[1-V_{k}(n)\right] .
$$

When $c \neq 1$, the solutions (46) become infinite either for $k \rightarrow \pm \infty$ or for $n \rightarrow \pm \infty$. The quantities $U_{k}(n), V_{k}(n)$ and thus also the conserved charges may remain finite, however.

2. $G L(n, \mathbb{R})$-models. We express an element $g \in G L(n, \mathbb{R})$ as $g= \pm e^{-q} s$ with real $q$ and $s \in S L(n, \mathbb{R})$. In the following we only discuss the case $\tau=0$ and $\lambda \neq 0$. The field equations (16) then split into the two parts,

$$
\ddot{q}_{k}=\frac{1}{n \lambda^{2}}\left[e^{q_{k-1}-q_{k}} \operatorname{tr}\left(s_{k-1}^{-1} s_{k}\right)-e^{q_{k}-q_{k+1}} \operatorname{tr}\left(s_{k}^{-1} s_{k+1}\right)\right]
$$

and

$$
\left(s_{k}^{-1} \dot{s}_{k}\right)^{\cdot}=\frac{1}{\lambda^{2}}\left(e^{q_{k}-q_{k+1}}\left[s_{k}^{-1} s_{k+1}-\frac{I}{n} \operatorname{tr}\left(s_{k}^{-1} s_{k+1}\right)\right]-e^{q_{k-1}-q_{k}}\left[s_{k-1}^{-1} s_{k}-\frac{I}{n} \operatorname{tr}\left(s_{k-1}^{-1} s_{k}\right)\right]\right)
$$

(where $I$ is the unit matrix). The first equation resembles that of the nonlinear Toda lattice to which it reduces for $s$ not depending on $k$.

3. A $G L(1, \mathbb{C})$-model. We write an element of $G L(1, \mathbb{C})$ in the form $g=e^{-q} e^{i \theta}$ with real $q$ and $\theta$. In this case the field equations for $\tau=0$ and $\lambda \neq 0$ read

$$
\begin{aligned}
& \ddot{q}_{k}=\frac{1}{\lambda^{2}}\left[e^{q_{k-1}-q_{k}} \cos \left(\theta_{k}-\theta_{k-1}\right)-e^{q_{k}-q_{k+1}} \cos \left(\theta_{k+1}-\theta_{k}\right)\right], \\
& \ddot{\theta}_{k}=\frac{1}{\lambda^{2}}\left[e^{q_{k-1}-q_{k}} \sin \left(\theta_{k}-\theta_{k-1}\right)-e^{q_{k}-q_{k+1}} \sin \left(\theta_{k+1}-\theta_{k}\right)\right] .
\end{aligned}
$$

A reduction to a $U(1)$-model by setting $q=0$ leads to a constraint. On the other hand, setting $\theta=0$ simply leads us back to the nonlinear Toda lattice.

4. A $G L(1, \mathbb{H})$-model. Here $\mathbb{H}$ denotes the quaternions. Again, we write an element in the form $g=e^{-q} u$ with real $q$ and $u=\alpha+\beta i+\gamma j+\delta k, u \bar{u}=\alpha^{2}+\beta^{2}+\gamma^{2}+\delta^{2}=1$. For $\tau=0$ and $\lambda \neq 0$ the field equations (16) take the form

$$
\begin{aligned}
\ddot{q}_{k} & =\frac{1}{2 \lambda^{2}}\left[e^{q_{k-1}-q_{k}}\left(\bar{u}_{k-1} u_{k}+\bar{u}_{k} u_{k-1}\right)-e^{q_{k}-q_{k+1}}\left(\bar{u}_{k} u_{k+1}+\bar{u}_{k+1} u_{k}\right)\right] \\
\left(\bar{u}_{k} \dot{u}_{k}\right)^{\cdot} & =\frac{1}{2 \lambda^{2}}\left[e^{q_{k-1}-q_{k}}\left(\bar{u}_{k-1} u_{k}-\bar{u}_{k} u_{k-1}\right)-e^{q_{k}-q_{k+1}}\left(\bar{u}_{k} u_{k+1}-\bar{u}_{k+1} u_{k}\right)\right] .
\end{aligned}
$$

Although $G L(1, \mathbb{H})$ is isomorphic with $S L(1, \mathbb{R}) \times U(2)$, if we had chosen the latter representation, some unpleasant constraints would have shown up.

5. The $O(n) \sigma$-model. Let $\mathbf{S} \in \mathbb{R}^{n}$ be a unit vector, $\mathbf{S} \bullet \mathbf{S}=1$. The matrix $g=I-2 P$ with $P=\mathbf{S} \otimes \mathbf{S}$ is orthogonal. We have $P^{2}=P$ and therefore $g^{-1}=g$. Hence

$$
A=2(P \mathrm{~d} P-\mathrm{d} P P)
$$


using $\mathrm{d} P=\mathrm{d} P^{2}=P \mathrm{~d} P+\mathrm{d} P P$. In terms of $\mathbf{S}$ we have

$$
A=2[\mathbf{S} \otimes \mathrm{d} \mathbf{S}-\mathrm{d} \mathbf{S} \otimes \mathbf{S}+2 \mathbf{S} \otimes(\mathbf{S} \bullet \mathrm{d} \mathbf{S}) \mathbf{S}]
$$

where we used $\mathrm{d} \mathbf{S} \bullet \mathbf{S}+\mathbf{S} \bullet \mathrm{d} \mathbf{S}=0$. P For $\tau \neq 0$ and $\lambda \neq 0$, we obtain

$$
\begin{aligned}
* A= & -2\left[\mathbf{S}^{-x} \otimes \partial_{-x} \mathbf{S}-\partial_{-x} \mathbf{S} \otimes \mathbf{S}+2\left(\mathbf{S}^{-x} \bullet \partial_{-x} \mathbf{S}\right) \mathbf{S}^{-x} \otimes \mathbf{S}\right] \mathrm{d} t \\
& -2\left[\mathbf{S}^{-t} \otimes \partial_{-t} \mathbf{S}-\partial_{-t} \mathbf{S} \otimes \mathbf{S}+2\left(\mathbf{S}^{-t} \bullet \partial_{-t} \mathbf{S}\right) \mathbf{S}^{-t} \otimes \mathbf{S}\right] \mathrm{d} x
\end{aligned}
$$

with $\mathbf{S}^{-t}(t, x):=\mathbf{S}(t-\tau, x)$ and a corresponding definition for $\mathbf{S}^{-x}$. The field equations $\mathrm{d} * A=0$ now take the form

$$
\begin{aligned}
& \partial_{+t}\left[\mathbf{S}^{-t} \otimes \partial_{-t} \mathbf{S}-\partial_{-t} \mathbf{S} \otimes \mathbf{S}+2\left(\mathbf{S}^{-t} \bullet \partial_{-t} \mathbf{S}\right) \mathbf{S}^{-t} \otimes \mathbf{S}\right]- \\
& \partial_{+x}\left[\mathbf{S}^{-x} \otimes \partial_{-x} \mathbf{S}-\partial_{-x} \mathbf{S} \otimes \mathbf{S}+2\left(\mathbf{S}^{-x} \bullet \partial_{-x} \mathbf{S}\right) \mathbf{S}^{-x} \otimes \mathbf{S}\right]=0
\end{aligned}
$$

In the limit $\tau, \lambda \rightarrow 0$ we get

$$
\mathbf{S} \otimes\left(\partial_{t}^{2}-\partial_{x}^{2}\right) \mathbf{S}=\left(\partial_{t}^{2}-\partial_{x}^{2}\right) \mathbf{S} \otimes \mathbf{S} .
$$

Acting on this equation from the left with $\mathbf{S} \bullet$ and using $\mathbf{S} \bullet \mathrm{d} \mathbf{S}=0$ (which holds in the case $\tau=\lambda=0$ ), we recover the field equations of the classical nonlinear $\sigma$-model on $\mathbb{R}^{2}$,

$$
\partial_{t}^{2} \mathbf{S}-\partial_{x}^{2} \mathbf{S}+\left(\partial_{t} \mathbf{S} \bullet \partial_{t} \mathbf{S}\right) \mathbf{S}-\left(\partial_{x} \mathbf{S} \bullet \partial_{x} \mathbf{S}\right) \mathbf{S}=0
$$

The conserved charges of this model were first obtained in [7] by means of inverse scattering methods. When $\tau=0$ and $\lambda \neq 0$, the field equations are

$$
\begin{aligned}
\mathbf{S}_{k} \otimes \partial_{t}^{2} \mathbf{S}_{k}-\partial_{t}^{2} \mathbf{S}_{k} \otimes \mathbf{S}_{k}= & \frac{1}{\lambda^{2}}\left[2\left(-\mathbf{S}_{k} \bullet \mathbf{S}_{k+1}\right) \mathbf{S}_{k} \otimes \mathbf{S}_{k+1}-2\left(\mathbf{S}_{k-1} \bullet \mathbf{S}_{k}\right) \mathbf{S}_{k-1} \otimes \mathbf{S}_{k}\right. \\
& \left.-\mathbf{S}_{k+1} \otimes \mathbf{S}_{k+1}+\mathbf{S}_{k-1} \otimes \mathbf{S}_{k-1}\right]
\end{aligned}
$$

in terms of $\mathbf{S}_{k}(t):=\mathbf{S}(t, \lambda k)$. Acting from the left' with $\mathbf{S}_{k} \bullet$ and using $\mathbf{S}_{k} \bullet \mathbf{S}_{k}=1$ (which implies $\left.\mathbf{S}_{k} \bullet \partial_{t}^{2} \mathbf{S}_{k}=-\partial_{t} \mathbf{S}_{k} \bullet \partial_{t} \mathbf{S}_{k}\right)$ we obtain

$$
\ddot{\mathbf{S}}_{k}+\left(\dot{\mathbf{S}}_{k} \bullet \dot{\mathbf{S}}_{k}\right) \mathbf{S}_{k}+\frac{1}{\lambda^{2}}\left[2\left(\mathbf{S}_{k} \bullet \mathbf{S}_{k-1}\right)^{2} \mathbf{S}_{k}-\left(\mathbf{S}_{k} \bullet \mathbf{S}_{k+1}\right) \mathbf{S}_{k+1}-\left(\mathbf{S}_{k} \bullet \mathbf{S}_{k-1}\right) \mathbf{S}_{k-1}\right]=0 .
$$

This does not exhaust the equations (62), however. In addition we have the constraint

$$
\begin{aligned}
\mathbf{S}_{k+1} \otimes \mathbf{S}_{k+1}-\mathbf{S}_{k-1} \otimes \mathbf{S}_{k-1} & +\left(\mathbf{S}_{k-1} \bullet \mathbf{S}_{k}\right) \mathbf{S}_{k} \otimes \mathbf{S}_{k-1}-\left(\mathbf{S}_{k} \bullet \mathbf{S}_{k+1}\right) \mathbf{S}_{k} \otimes \mathbf{S}_{k+1} \\
& +\left(\mathbf{S}_{k-1} \bullet \mathbf{S}_{k}\right) \mathbf{S}_{k-1} \otimes \mathbf{S}_{k}-\left(\mathbf{S}_{k} \bullet \mathbf{S}_{k+1}\right) \mathbf{S}_{k+1} \otimes \mathbf{S}_{k}=0 .
\end{aligned}
$$

The problem is that this constraint is not automatically respected by (63) so that differentiation with respect to time generates additional equations of motion. The conclusion is that the equations governing our lattice $O(n) \sigma$-model are not 'good' equations. There are at least simple exact solutions like those given by

$$
\mathbf{S}_{k} \bullet \mathbf{S}_{k+1}=0, \quad \mathbf{S}_{k+2}= \pm \mathbf{S}_{k}, \quad \ddot{\mathbf{S}}_{k}+\left(\dot{\mathbf{S}}_{k} \bullet \dot{\mathbf{S}}_{k}\right) \mathbf{S}_{k}=0
$$

\footnotetext{
${ }^{5}$ Note that (for $\tau \neq 0$ or $\lambda \neq 0$ ) the noncommutativity between functions and differentials leads to $\mathbf{S} \bullet \mathrm{d} \mathbf{S} \neq 0$, in general.

${ }^{6}$ Acting from the right with $\bullet \mathbf{S}_{k}$ yields the same equation but with $\mathbf{S}_{k+1}$ replaced by $\mathbf{S}_{k-1}$ in the quadratic term on the rhs.
} 
The appearance of a constraint, as in our last example, is a rather general feature which can be understood as follows. The equation $\mathrm{d} *\left(g^{-1} \mathrm{~d} g\right)=0$, where $g$ has values in a group, takes the following form for $\tau=0$ and $\lambda \neq 0$,

$$
\partial_{t}\left(g_{k}^{-1} \partial_{t} g_{k}\right)=\frac{1}{\lambda^{2}}\left(g_{k}^{-1} g_{k+1}-g_{k-1}^{-1} g_{k}\right)
$$

Whereas the lhs is in the Lie algebra of the group, the rhs lives in the group algebra. The deviation of the group algebra from the Lie algebra results in constraints. In case of the orthogonal group $O(n)$, the lhs of the last equation is an antisymmetric matrix, but this property is not shared by the rhs. The symmetric part of the matrix equation is then a constraint. This problem does not appear for $G L(n)$-models. In case of the $O(n)$-model, one may take into consideration a simultaneous deformation of $\mathbf{S} \bullet \mathbf{S}=1$ as an attempt to get rid of the constraints.

\section{Lax pairs for the chiral models}

In this section we construct Lax pairs for our chiral models. This establishes contact with other formulations of integrable models (see [6], for example). The starting point is the equation $* \mathrm{~d} \chi=\gamma D \chi$ derived in section 3 .

1) $M=\mathbb{R}^{2}$. Evaluation of (32) leads to

$$
\partial_{t} \chi=-\gamma\left[\partial_{x} \chi+\left(g^{-1} \partial_{x} g\right) \chi\right], \quad \partial_{x} \chi=-\gamma\left[\partial_{t} \chi+\left(g^{-1} \partial_{t} g\right) \chi\right] .
$$

Solving this system for the partial derivatives of $\chi$, we obtain

$$
\partial_{x} \chi=L \chi, \quad \partial_{t} \chi=M \chi
$$

with

$$
L(t, x ; \gamma)=\frac{\gamma}{1-\gamma^{2}} g^{-1}\left(-\partial_{x} g+\gamma \partial_{t} g\right), \quad M(t, x ; \gamma)=\frac{\gamma}{1-\gamma^{2}} g^{-1}\left(\gamma \partial_{x} g-\partial_{t} g\right)
$$

In terms of $L$ and $M$ the integrability conditions for the system (68), which are the field equations, read

$$
\partial_{t} L-\partial_{x} M+[L, M]=0
$$

2) $M=\mathbb{R} \times \mathbb{Z}$. In this case (32) yields

$$
\dot{\chi}_{k}=\frac{\gamma}{\lambda}\left[g_{k}^{-1} g_{k+1} \chi_{k+1}-\chi_{k}\right], \quad \chi_{k}-\chi_{k-1}=-\gamma \lambda\left[\dot{\chi}_{k}+g_{k}^{-1} \dot{g}_{k} \chi_{k}\right] .
$$

Introducing $\psi_{k}:=\chi_{k-1}, \phi_{k}:=\gamma g_{k} \chi_{k}$ and $\xi_{k}:=\left(\phi_{k}, \psi_{k}\right)^{T}$, we obtain

$$
\xi_{k+1}=L_{k} \xi_{k}, \quad \dot{\xi}_{k}=M_{k} \xi_{k}
$$

with

$$
L_{k}(t ; \gamma)=\gamma^{-1}\left(\begin{array}{cc}
\left(\gamma^{-1}+\gamma\right) I+\lambda \dot{g}_{k} g_{k}^{-1} & -g_{k} \\
g_{k}^{-1} & 0
\end{array}\right)
$$


and

$$
M_{k}(t ; \gamma)=\lambda^{-1}\left(\begin{array}{cc}
-\gamma^{-1} I & g_{k} \\
-g_{k-1}^{-1} & \gamma I
\end{array}\right)
$$

The field equations of the chiral model are now obtained as integrability conditions of the linear system $(72)$ in the form

$$
\partial_{t} L+L_{k} M_{k}-M_{k+1} L_{k}=0
$$

3) $M=\mathbb{Z}^{2}$. Now (32) leads to

$$
\begin{aligned}
\chi_{k}(n)-\chi_{k}(n-1) & =-\frac{\gamma}{c}\left[g_{k}(n)^{-1} g_{k+1}(n) \chi_{k+1}(n)-\chi_{k}(n)\right], \\
\chi_{k}(n)-\chi_{k-1}(n) & =-\gamma c\left[g_{k}(n)^{-1} g_{k}(n+1) \chi_{k}(n+1)-\chi_{k}(n)\right]
\end{aligned}
$$

where $c:=\lambda / \tau$. Let us introduce $\psi_{k}(n):=\chi_{k-1}(n-1), \phi_{k}(n):=\gamma g_{k}(n) \chi_{k}(n)$ and $\xi_{k}(n):=\left(\phi_{k}(n), \psi_{k}(n)\right)^{T}$. Then

$$
\xi_{k+1}(n)=L_{k}(n) \xi_{k}(n), \quad \xi_{k}(n+1)=M_{k}(n) \xi_{k}(n)
$$

with

$$
L_{k}(n ; \gamma)=(1-\gamma c)^{-1} \hat{L}_{k}(n ; \beta), \quad M_{k}(n ; \gamma)=(c-\gamma)^{-1} c^{-1} \hat{M}_{k}(n ; \beta)
$$

where

$$
\begin{aligned}
\hat{L}_{k}(n ; \beta) & =\left(\begin{array}{cc}
\beta I-c^{2} g_{k}(n)\left[g_{k}(n-1)\right]^{-1} & c g_{k}(n) \\
-c\left[g_{k}(n-1)\right]^{-1} & I
\end{array}\right) \\
\hat{M}_{k}(n ; \beta) & =\left(\begin{array}{cc}
\beta I-g_{k}(n)\left[g_{k-1}(n)\right]^{-1} & c g_{k}(n) \\
-c\left[g_{k-1}(n)\right]^{-1} & c^{2} I
\end{array}\right)
\end{aligned}
$$

and $\beta=1-c\left(\gamma+\gamma^{-1}\right)+c^{2}$. Now the field equations are obtained as

$$
\hat{L}_{k}(n+1) \hat{M}_{k}(n)=\hat{M}_{k+1}(n) \hat{L}_{k}(n)
$$

(suppressing the argument $\beta$ ). The matrices $\hat{L}_{k}$ and $\hat{M}_{k}$ are invertible if $\beta \neq 0$.

\section{Bäcklund transformations for the chiral models}

If $g$ is an exact solution of the field equation for a chiral model, then also $h$ if

$$
h^{-1} \mathrm{~d} h-g^{-1} \mathrm{~d} g=* \mathrm{~d} F
$$

with a $\mathcal{B}$-valued function $F$. A suitable choice for $F$ is essential for this relation to be useful for generating new solutions from given solutions. The usual continuum $\left(M=\mathbb{R}^{2}\right)$ Bäcklund transformation is obtained for $F=\beta h^{-1} g$ where $\beta$ is the spectral parameter (see [8], for example). For the nonlinear Toda lattice the relation

$$
h_{k}^{-1} \mathrm{~d} h_{k}-g_{k}^{-1} \mathrm{~d} g_{k}=\beta * \mathrm{~d}\left(h_{k}^{-1} g_{k+1}\right)
$$


reproduces the corresponding formulas in [4]. After some manipulations we find, for $\tau=0$ and $\lambda \neq 0$,

$$
\begin{aligned}
g_{k}^{-1} \dot{g}_{k} & =\frac{1}{\lambda}\left(\beta h_{k-1}^{-1} g_{k}+\frac{1}{\beta} g_{k}^{-1} h_{k}+\alpha I\right) \\
h_{k}^{-1} \dot{h}_{k} & =\frac{1}{\lambda}\left(\beta h_{k}^{-1} g_{k+1}+\frac{1}{\beta} g_{k}^{-1} h_{k}+\alpha I\right)
\end{aligned}
$$

where $\alpha$ is a constant of integration. Surprisingly, written in this way it generalizes to a Bäcklund transformation for the general chiral model on $M=\mathbb{R} \times \mathbb{Z}$. However, for a noncommutative group $\mathcal{B}^{*}$ the above set of equations is no longer equivalent to (82), though there must be some $F$ so that (81) is satisfied.

Now we turn to the remaining case $M=\mathbb{Z}^{2}$. Here we proceed in a different way. For an invertible matrix

$$
\mathcal{M}=\left(\begin{array}{ll}
a & b \\
c & d
\end{array}\right)
$$

with entries $a, b, c, d \in \mathcal{B}$ and for $z \in \mathcal{B}$ we define $\mathcal{P}(\mathcal{M}) z:=(a z+b)(c z+d)^{-1}$. Using $\mathcal{P}(\mathcal{M}) \mathcal{P}\left(\mathcal{M}^{\prime}\right)=\mathcal{P}\left(\mathcal{M} \mathcal{M}^{\prime}\right)$ the field equations in the form (80) can now be rewritten as

$$
\mathcal{P}\left(\hat{L}_{k}(n+1)\right) \mathcal{P}\left(\hat{M}_{k}(n)\right)=\mathcal{P}\left(\hat{M}_{k+1}(n)\right) \mathcal{P}\left(\hat{L}_{k}(n)\right)
$$

For $h_{k}(n) \in \mathcal{B}^{*}$ we define

$$
h_{k+1}(n)=\mathcal{P}\left(\hat{L}_{k}(n)\right) h_{k}(n), \quad h_{k}(n+1)=\mathcal{P}\left(\hat{M}_{k}(n)\right) h_{k}(n) .
$$

The field equations for $g_{k}(n)$ are now recovered as integrability conditions of this system, i.e., by calculating $h_{k+1}(n+1)$ from both of the last two equations. The latter can be written in the form

$$
\begin{aligned}
h_{k+1}(n) & =c g_{k}(n)+\beta\left(c\left[g_{k}(n-1)\right]^{-1}-\left[h_{k}(n)\right]^{-1}\right)^{-1} \\
c h_{k}(n+1) & =g_{k}(n)+\beta\left(\left[g_{k-1}(n)\right]^{-1}-c\left[h_{k}(n)\right]^{-1}\right)^{-1}
\end{aligned}
$$

or, equivalently,

$$
\begin{aligned}
g_{k+1}(n) & =c h_{k+1}(n+1)+\beta\left(c\left[h_{k+1}(n)\right]^{-1}-\left[g_{k}(n)\right]^{-1}\right)^{-1} \\
c g_{k}(n+1) & =h_{k+1}(n+1)+\beta\left(\left[h_{k}(n+1)\right]^{-1}-c\left[g_{k}(n)\right]^{-1}\right)^{-1} .
\end{aligned}
$$

The last set of equations shows that the field equations for $g_{k}(n)$ are also obtained by calculating $g_{k+1}(n+1)$ from both equations and comparing the results. As a consequence, if $g_{k}(n)$ is a solution, then also $h_{k}(n)$. Hence (87) defines a Bäcklund transformation. Applied to the left movers (46) of the Toda model on $\mathbb{Z}^{2}$ and writing $h_{k}(n)=e^{-q_{k}^{\prime}(n)}$, the transformation leads to $q_{k}^{\prime}(n+1)=c^{-2} q_{k+1}^{\prime}(n)$ (together with a constraint on initial values) and thus again to left movers. 


\section{Conclusions}

We have presented a general method for integrable discretizations of two-dimensional chiral models via deformations of the ordinary differential calculus. As an example, the nonlinear Toda lattice is obtained in this way from the linear wave equation. It may be regarded as a $G L(1, \mathbb{R})$ chiral model on $\mathbb{R} \times \mathbb{Z}$. More generally, our method works well in particular for $G L(n, \mathbb{R})$ and $G L(n, \mathbb{C})$ models. In case of a chiral model for which the group algebra differs from the Lie algebra, the resulting discretized model in general suffers from unpleasant constraints, however. This has been demonstrated for the $O(n) \sigma$-model. Perhaps there are modifications of our formalism which can improve such models.

The general formalism presented here also works for corresponding deformations of the differential calculus on $\mathbb{R} \times S^{1}$ to calculi on a periodic (space) lattice. In that case, however, the first cohomology group is no longer trivial and (22) must no longer hold. But if the continuum chiral model is integrable, then also its deformations. Furthermore, one may consider other (and in particular curved) metrics on the underlying two-dimensional space. In fact, the possibilities to generalize the formalism developped in this work extend much beyond what we have mentioned so far. The deformed differential calculus of section 2 may be replaced by other differential calculi with a two-dimensional space of 1-forms (see [9] for candidates). All we need is a suitable generalization of the $*$-operator. It is then possible to generalize the (continuum) definition of a chiral model. This is what we have done for a restricted class of differential calculi. It turned out that the nonlinear Toda lattice belongs to the corresponding extended class of chiral models. It remains to be seen whether other integrable models can also be understood as generalized chiral models.

Acknowledgment. F M-H thanks M. Bordemann for a motivating discussion.

\section{References}

[1] A. Dimakis, F. Müller-Hoissen and T. Striker, "From continuum to lattice theory via deformation of the differential calculus", Phys. Lett. 300B (1993) 141; "Noncommutative differential calculus and lattice gauge theory", J. Phys. A 26 (1993) 1927; A. Dimakis and F. Müller-Hoissen, "Quantum mechanics on a lattice and $q$-deformations", Phys. Lett. 295B (1992) 242

[2] A.M. Perelomov, "Chiral models: geometrical aspects", Phys. Rep. 146 (1987) 135

[3] E. Brezin, C. Itzykson, J. Zinn-Justin and J.-B. Zuber, " Remarks about the existence of nonlocal charges in two-dimensional models", Phys. Lett. 82B (1979) 442

[4] M. Toda, Theory of Nonlinear Lattices (Springer, 1989)

[5] Yu.B. Suris, "Discrete time generalized Toda lattices: complete integrability and relation with relativistic Toda lattices", Phys. Lett. A145 (1990) 113

[6] L.D. Faddeev and L.A. Takhtajan, Hamiltonian Methods in the Theory of Solitons (Springer, 1987)

[7] M. Lüscher and K. Pohlmeyer, "Scattering of massless lumps and non-local charges in the two-dimensional classical non-linear $\sigma$-model", Nucl. Phys. B137 (1978) 46 
[8] A.T. Ogielski, M.K. Prasad and A. Sinha, "Bäcklund transformations and local conservation laws for principal chiral fields", Phys. Lett. 91B (1980) 387

[9] H.C. Baehr, A. Dimakis and F. Müller-Hoissen, "Differential calculi on commutative algebras", J. Phys. A 28 (1995) 3197 